\title{
Überlegungen zu einer ungewöhnlichen Schreibung des ägyptischen „3bnn“ - Vogels
}

\author{
Stefan Bojowald \\ Universität Bonn
}

\begin{abstract}
In this article, a peculiar writing of the Egyptian " $3 b n n$ " - bird is tackled. The "3bnn" - bird belongs to those species that are unidentified until now. In oBM EA 50727, recto 2, this birdname has received the determinative of "Cow skin", for which an explanation is searched. Needless to say, that this determinative is normally reserved for mammals. It will be shown however that, as an exception, this determinative can be used for insects, amphibians, reptiles, spiders, locusts and worms, too. Several examples will be collected. In the case under consideration the crucial point will be if this determinative can be used for birds as well. The question can be answered in the affirmative. In the Demotic pBerlin P 8932 vso, 6, the determinative of the "Cow skin" is combined with the "gs" - fowl. Therefore, the "3bnn" - bird represents the second example of this special use.
\end{abstract}

Keywords: Egyptian bird name, determinative of "Cow skin" in general, determinative of "Cow skin" after amphibians, reptiles, insects, spiders, locusts and worms in special, determinative of "Cow 
skin" after bird-names, explanation for the determinative of "Cow skin" after the "3bnn" - bird.

In der Reihe der ägyptischen Vogelbezeichnungen ist auch der Name des „3bnn“" ${ }^{1}$ - Vogels zu nennen, der an mehreren Stellen nachgewiesen ist. Die Frage nach dem ursprünglichen Besitzer dieses Namens konnte bisher noch nicht endgültig geklärt werden. Wenn jedoch Hannig ${ }^{2}$ Recht hat, könnte es sich dabei um einen flugunfähigen Vogel gehandelt haben. Das in Edfou I, 343, 5 nach „3bnn“" gewählte phönixartige Determinativ eignet sich dagegen nur bedingt zur Vogelbestimmung, da es in erster Linie durch den ähnlichen Klang des Wortes mit „bnw“ „Phönix“ inspiriert sein dürfte, der schon von Brose ${ }^{3}$ angesprochen worden ist.

In den folgenden Zeilen wird ein Aspekt bezüglich dieses Vogelnamens zur Sprache kommen, der bislang zu sehr in den Hintergrund getreten ist. Im konkreten Fall wird es um eine besonders eigenwillige Schreibung dieses Wortes gehen.

In oBM EA 50727, recto 2, ist hinter den Vogelnamen $* 1 \pi=4^{4}$ das Determinativ des „Tierfelles“ gesetzt worden, das noch immer nicht zufriedenstellend erklärt ist. In der jüngsten Bearbeitung durch Brose ist jedenfalls auf einen Kommentar verzichtet worden. Die Feststellung, dass dieses Determinativ in der Hauptsache für

\footnotetext{
${ }^{1}$ WB I, 8, 6; W. Spiegelberg, Hieratic Ostraka \& Papyri, found by J. E. Quibell in the Ramesseum, 1895-6, BSA 2 Extra (1989), XLIII; A. H. Gardiner, Ancient Egyptian Onomastica, Text, Volume II (1947), *256; J. Osing, Die Nominalbildung des Ägyptischen, Textband, SDAIK 3A (1976), 298; L. H. Lesko, A dictionary of Late Egyptian, Volume I (1982), 6; H. S. Smith/W. J. Tait, Saqqara Demotic Papyri I (P. Dem. Saq. I) Texts from Excavations 7 (1983), 206 (Kontext zerstört!); R. Hannig, Großes Handwörterbuch Ägyptisch - Deutsch (2800-950 v. Chr.), 4. überarbeitete Auflage (2006), 6; M. Brose, SAK 38 (2009), 65. Der Vogelname „3bnn“ ist von J. Osing, Die Nominalbildung des Ägyptischen, Textband, SDAIK 3A (1976), 298, als Diminutiv aufgefasst worden, wobei er sich auf den Bildungstyp mit geminiertem dritten Radikal gestützt hat. Die bei J. Osing, Die Nominalbildung des Ägyptischen, Textband, SDAIK 3A (1976), 298, vorgenommene Herleitung des Vogelnamens von der Wurzel , $3 b n^{\prime \prime}$ „drehen“ muss jedoch als fraglich eingestuft werden.

${ }^{2}$ R. Hannig, Großes Handwörterbuch Ägyptisch - Deutsch (2800-950 v. Chr.), 4. überarbeitete Auflage (2006), 6.

${ }^{3}$ M. Brose, SAK 38 (2009), 65.

${ }^{4}$ R. J. Demarée, Ramesside Ostraca (2002), Pl. 122; M. Brose, SAK 38 (2009), 59.
} 
Säugetiere verwendet worden ist, dürfte fast schon trivial sein. Die Tatsache ist ebenfalls nicht neu, dass diese Regel in Ausnahmefällen auch durchbrochen werden konnte. In diesem Rahmen könnte auf den Gebrauch dieses Determinatives nach Spinnentieren wie der Tarantel und dem ${ }^{6}$ - Skorpion verwiesen werden. Das Determinativ ist auch bei Insektenbezeichnungen ${ }^{7}$ wie dem $\square=\mathbb{A} 4 f=7$ ${ }^{8}$ - Käfer und dem ${ }^{1} 49^{9}$ - Floh verwendet worden. Der Gebrauch des Determinatives hat sich außerdem auf Heuschrecken wie die $\triangle \mathbb{A} 4<-10$

${ }^{5}$ C. E. Sander-Hansen, Die Texte der Metternichstele, AnalAeg 7 (1956), 43; S. Sauneron, Un traité égyptien d'Ophiologie, Papyrus du Brooklyn Museum $\mathrm{N}^{0}$ 47.218.48 et .85, BibGen 11 (1989), 44. Zur Bestimmung von ,intš als Tarantel vgl. J. F. Borghouts, Ancient Egyptian Magical Texts, Nisaba 9 (1978), 69 Nr. 93/94 Nr. 143; R. Hannig, Großes Handwörterbuch Ägyptisch - Deutsch (2800-950 v. Chr.), (2006), 91

${ }^{6}$ J. Cerny, Ostraca Hiératiques, Catalogue Général des Antiquités Égyptiennes du Musée du Caire, $\mathrm{N}^{\mathrm{os}} 25501$ - 25832, Tome Premièr (1935), 17*/19*/24*; K. JansenWinkeln, Inschriften der Spätzeit, Teil II: Die 22. - 24. Dynastie (2007), 459. Zur Ableitung des Wortes „wh`.t" „Skorpion“ von der Wurzel „wh" „stechen“ vgl. E. Edel, Zu den Inschriften auf den Jahreszeitenreliefs der „Weltenkammer“ aus dem Sonnenheiligtum des Niuserre, Nachrichten der Akademie der Wissenschaften in Göttingen, 1. Philologisch - Historische Klasse, II. Teil, (1963), 162; I. GamerWallert, Fische und Fischkulte im Alten Ägypten, ÄgAb 21 (1970), 33; J. Osing, Die Nominalbildung des Ägyptischen, Textband, SDAIK 3A (1976), 120. Beachte das Wortspiel zwischen „wh؟.t" „Skorpion“ und „wh" „ablassen“ bei C. E. SanderHansen, Die Texte der Metternichstele, AnalAeg 7 (1956), 45-73.

${ }^{7} \mathrm{Zu}$ einem unklaren Fall des Determinatives des „Tierfelles” nach einer möglichen Insektenbezeichnung vgl. L. Keimer, ASAE 33 (1933), 195-196.

${ }^{8}$ W. R. Dawson, JEA 20 (1934), 187; J. Osing, StudAeg 14 (1992), 475; Chr. Leitz, Hieratic Papyri in the British Museum VII, Magical and medical papyri of the New Kingdom (1999), 59 n. 62. Das „'pš3ii.t“ - Insekt muss bei I. Munro, Der TotenbuchPapyrus des Hohenpriesters Pa-nedjem II. (pLondon BM 10793/pCampbell), HAT 3 (1996), 25, als ein dem Toten gefährlich gewordener Schädling abgewehrt werden. Der Name des Kerbtieres ist bei D. Meeks, Les emprunts Égyptiens aux langues sémitiques durant le Nouvel Empire et la Troisième Période Intermediaire. Les aléas

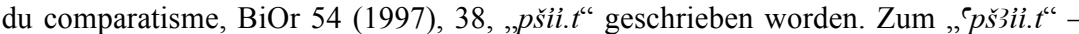
Käfer vgl. auch W. Westendorf, Handbuch der altägyptischen Medizin, 1. Band, Handbuch der Orientalistik, Erste Abteilung, Der Nahe und Mittlere Osten, 36. Band (1999), 496.

${ }^{9}$ Ward, CdE 71 (1996), Fasc. 141, 45. Zum „,p(w)ïi“ - Floh vgl. F. Calice, Grundlagen der ägyptisch-semitischen Wortvergleichung, Eine kritische Diskussion des bisherigen Vergleichsmaterials, Beihefte zur Wiener Zeitschrift für die Kunde des Morgenlandes, 1. Heft (1936), 147.

${ }^{10}$ W. Westendorf, Handbuch der altägyptischen Medizin, 1. Band, Handbuch der Orientalistik, Erste Abteilung, Der Nahe und Mittlere Osten, 36. Band (1999), 508. Zu diesem Tier vgl. auch J. Osing, StudAeg 14 (1992), 476; W. Westendorf, in: M. 
- Heuschrecke (?) erstreckt. In gleicher Weise konnte es nach Reptilbezeichnungen wie der $\underbrace{11}-$ Schlange, der ${ }^{12}-$ Eidechse, dem -- Krokodil und der $=\mathbb{1}^{13}$ - Schildkröte gesetzt werden. Das Determinativ ist außerdem mit Amphibien wie dem $\square-0^{15}$ Wassermolch, dem $\ell_{-}{ }^{16}$ - Frosch und dem $\Delta \mathbb{R}=\mathbb{R}^{17}$ - Frosch (?) verbunden worden.

Minas/J. Zeidler (Hrsg.), Aspekte spätägyptischer Kultur, Festschrift für Erich Winter zum 65. Geburtstag, AegTrev VII (1994), 265; S. Sauneron, Une traité égyptien d'Ophiologie, Papyrus du Brooklyn Museum No 47.218.48 et .85, BibGen 11 (1989), 55; J. Osing, Hieratische Papyri aus Tebtunis I, The Carlsberg Papyri 2, CNI Publications 17 (1998), 123 j; Chr. Leitz, Tagewählerei, Das Buch h3.t nhh ph.wi d.t und verwandte Texte, Textband, ÄgAb 55 (1994), 43; Chr. Leitz, Magical and Medical Papyri of the New Kingdom, Hieratic Papyri in the British Museum VII (1999), 59 n. 63.

${ }^{11}$ Chr. Leitz, Tagewählerei, Das Buch h3.t nhh ph.wi d.t und verwandte Texte, Textband, ÄgAb 55 (1994), 46. Die oben vorgeschlagene Erklärung des Determinatives des "Tierfelles“ bei der " $n h h^{\text {“ }}$ - Schlange mit dessen Verwendung bei Reptilien scheint den Kern der Sache besser als eine mögliche Übemahme von der "`nh" - Ziege zu treffen.

${ }_{12} \mathrm{~S}$. Sauneron, Une traité égyptien d'Ophiologie, Papyrus du Brooklyn Museum N ${ }^{\circ}$ 47.218.48 et .85, BibGen 11 (1989), 14/17 (?).

${ }^{13}$ A. de Buck, The Egyptian Coffin Texts VI. Texts of Spells 472-786, The University of Chicago Oriental Institute Publications Volume LXXXI (1956), VI 193a; I. E. S. Edwards, Hieratic Papyri in the British Museum, Fourth Series: Oracular amuletic decrees of the Late New Kingdom, Vol. I, Text (1960), 75.

${ }^{14}$ WB IV, 557, Belegschreibungen; S. Sauneron, Un traité égyptien d'Ophiologie, Papyrus du Brooklyn Museum No 47.218.48 et .85, BibGen 11 (1989), 103. Die , $\check{s} t w$ “ - Schildkrote hat in WB IV, 557, 6, der mit dem Logogramm der Schildkröte geschriebenen ,̌stw" - Pflanze zu ihrem Namen verholfen. In der Zwischenzeit hat sich diese Lesung jedoch als Irrtum herausgestellt. Wie S. Sauneron, BIFAO 64 (1966), 1ff; Charpentier, Recueil de matériaux épigraphiques relatifs a la botanique de l'Égypte Antique (1981), 702, nachgewiesen haben, muss die Schreibung „mnh““ „Papyrus“" gelesen werden.

${ }^{15} \mathrm{~S}$. Sauneron, Une traité égyptien d'Ophiologie, Papyrus du Brooklyn Museum $\mathrm{N}^{\circ}$ 47.218.48 et .85, BibGen 11 (1989), 114. Zum „'pnn.t" - Wassermolch vgl. J. Osing, Die Nominalbildung des Ägyptischen, Textband, SDAIK 3A (1976), 298. Im Gegensatz dazu hat sich Chr. Leitz, Hieratic Papyri in the British Museum VII, Magical and Medical Papyri of the New Kingdom (1999), 7 n. 37, skeptisch zur Bedeutung „Wassersalamander (sic!)“ geäußert.

${ }^{16}$ Z. Zaba, The Rock Inscriptions of Lower Nubia (Czechoslovak Concession), Charles University of Prague, Czechoslovak Institute of Egyptogy in Prague and in Cairo Publications, Volume I (1974), 109.

${ }^{17}$ H. Ranke, ZAS 60 (1925), S. 80 n. 1. Zur onomatopoetischen Bildung des „krr.w“ Frosches vgl. J. Osing, Die Nominalbildung des Ägyptischen, Anmerkungen und Indices, SDAIK 3B (1976), 354. 
Wenn dieses Determinativ demnach bei so vielen Nichtsaugetieren stehen kann, lautet die entscheidende Frage, ob dies auch bei Vögeln möglich ist. Im Falle einer positiven Antwort könnte das Rätsel um das Determinativ des „Tierfelles“ beim „3bnn“ - Vogel rasch gelöst werden. Wie es aussieht, kann die zu Beginn gestellte Frage eindeutig bejaht werden. Im demotischen pBerlin P 8932 vso, 6 ist das Determinativ des „Tierfelles“ hinter das „gšc ${ }^{\text {(18 }}$ - Geflügel getreten. Obwohl bisher nur dieses eine Beispiel bekannt gewesen ist, steht die gewünschte Information damit einwandfrei fest. Das mit dem „Tierfell“ determinierte „mśr. $t^{\star 19}$ - Tier sollte besser beiseite gelassen werden, da der dortige Kontext aufgrund zahlreicher Zer-störungen keine Aussagen darüber zulässt, ob hier ein weiterer Beleg für den in pSallier IV vso 2, 4 bezeugten ,mśr.t" - Vogel vorliegt. Der Gebrauch des Determinatives des „Tierfelles“ bei Vögeln darf jedoch auf Basis zweier sicherer Beispiele als bewiesen gelten.

\footnotetext{
${ }^{18}$ S. L. Lippert/M. Schentuleit, Quittungen, Demotische Dokumente aus Dime, Band II (2006), 107 (freundlicher Hinweis M. Schentuleit). Zur ,g3š“- Gans/Ente vgl. auch J. Osing, Hieratische Papyri aus Tebtunis I, Text, The Carlsberg Papyri 2, CNI 17 (1998), 128. Das Wort „gডّ“ hat in sekundärer Verwendung Zugvögel bezeichnet, worauf bereits E. Edel, LÄ VI, 1424, s. v. Zugvögel, aufmerksam gemacht hat. Zu den , $g(3) \breve{s}^{“ c}-$ Zugvögeln vgl. A. Piankoff, The Litany of Re, Egyptian Religious Texts and representations, Bollingen Series XL 4 (1964), 29; H. Goedicke, The Report of Wenamun, The Johns Hopkins Near Eastern Studies 6 (1975), 120.

${ }^{19}$ A. H. Gardiner, Hieratic Papyri in the British Museum, Third Series, Chester Beatty Gift, Volume II, Plates (1935), Pl. 21, 44.
} 
